\title{
A Standardized Power Quality Information Platform for Multisource Information Fusion
}

\author{
JIA Ya Jun ${ }^{1, a}$, ZHANG Hong Wei ${ }^{1, a}$, CUI Xiao ${ }^{1, b}, \mathrm{JIA} \mathrm{Xu}^{1, c}$, \\ and YUAN Jin feng ${ }^{2, d^{*}}$ \\ ${ }^{1}$ State Grid Qingdao Power Supply Company, Liujiaxia Road, Shinan District, Qingdao, Shandong, \\ China \\ ${ }^{2}$ Titans Building, Shihua West Road, Zhuhai, Guangdong, China \\ a 18653205957@163.com, b24702989@sina.com, c13156368668@163.coM, \\ d11808982@qq.com
}

Keywords: power quality; standardized information platform; multisource information fusion; IEC 61850; IEC 61970; Web Service

Abstract. At present the nonstandard power quality monitoring systems have caused many problems such as high construction and maintenance cost and poor interoperability. In order to implement a standardized power quality information platform for multisource information fusion, IEC 61970 and IEC 61850 are designed as the fundamental methods for structuring the PQIP architecture. The integration solution of IEC 61970 and IEC 61850 are researched to enhance the commonality of PQIP. The use of GID standardized interfaces, the CIM standardized data model and Web Service enables the PQIP to realize functional and data interaction with other software systems.

\section{Introduction}

Power Quality (PQ) has become an increasing concern for both utilities and commercial and industrial electrical power customers. In recent years there has been an increase in the number of system loads that are sensitive to PQ problems, as well as an increase in the number of system loads that are themselves a source of poor PQ (such as harmonics caused by variable speed motor drives). Loads such as computers, process controls, and communications equipment are more sensitive to PQ variations due to system disturbances than in the past ${ }^{[1]}$.

Along with the development of information communication technology, some PQ Monitoring Systems (PQMS) have been implemented in power supply enterprise. Compared with the existing software systems such as SCADA, relay protection information system and metering information system in power supply enterprise, PQMS needs to handle more types of monitoring data, but most PQMS is nonstandard because of the PQ Monitoring Devices (PQMD) accessing to the systems, the network it exists, the operating platform and development environment are different ${ }^{[2-3]}$. The nonstandard systems can cause many problems such as the high construction cost for both the PQSM and PQMD providers, the PQ monitoring data can't be used by other systems and it's also difficult for PQSM to take advantage of the other system's usefully data without a complicated and user-defined interface program which will increase the complexity of the maintenance ${ }^{[1]}$.

In order to overcome these shortcomings caused by the nonstandard systems, in this paper a standardized Power Quality Information Platform (PQIP) for multisource information fusion is proposed. The premise of multisource information fusion is standardization. The proposed standardized remodeling solution is based on the IEC (International Electrotechnical Commission) international standards, in particular, IEC 61850 and IEC 61970 standards. The IEC 61970 remodeling solution for PQMS is based on web services technology that enables the typical advantages of an open-source framework, such as applications decoupling, applications integration, reduction of upgrading costs, reuse of existing infrastructures, and ease of development. The remodeling solution for PQMD is based on IEC 61850 to implement data sharing and real-time data transmission. By adopting the proposed standardized remodeling solution, the PQIP can interchange 
power grid model and data with other systems, and different kinds of PQMD can access the PQIP by using uniform communication protocol which can reduce the construction cost and development time, and take full advantage of PQ monitoring data.

\section{The Integration of IEC 61970 and IEC 61850 Standards}

The main objective of the IEC 61970 and IEC 61850 standards is the minimization of the effort required to perform integration and achievement of flexible software infrastructure such as PQIP. The use of IEC standards guarantees interoperability between applications and their data models and between communication protocols. The key aspect is the introduction of standardized data models of IEC 61850 for standards-compliant devices.

In this paper, the basic idea is to use the generic interface definition (GID) to provide standardized interfaces and the common information model (CIM) to handle standardized data models when the information interchange is needed between PQIP and other systems. Hence the PQIP is modeled based on the IEC 61970. The communication between PQMD and PQIP is based on IEC 61850 which is in order to set up a uniform communication protocol for both PQMD and PQIP providers. In order to realize the data transmission from the PQMD based on IEC 61850 to the PQIP based on IEC 61970, the integrated solution of IEC 61970 and IEC 61850 for the PQ monitoring is proposed in this paper. The diagram of the integrated solution is shown in Figure 1.

\begin{tabular}{|c|c|c|}
\hline PQIP/EMS/SCADA & \multirow{3}{*}{$\begin{array}{c}\text { IEC } 61850 \\
\text { MMS }\end{array}$} & \\
\hline \multirow{2}{*}{$\begin{array}{l}\text { IEC } 61850 \text { and IEC } 61970 \text { model } \\
\text { mapping and integration inside the PQIP }\end{array}$} & & Substation Automation System \\
\hline & & \\
\hline $\begin{array}{l}\text { The IEC61850 Client: } \\
\text { Communicate with the power }\end{array}$ & $\sqrt{\text { IEC 61850-90-2 }}$ & $\begin{array}{l}\text { The IEC } 61850 \text { Server: } \\
\text { inside the PQ monitoring devices }\end{array}$ \\
\hline
\end{tabular}

Fig. 1 The diagram of the integrated solution of IEC 61970 and IEC 61850 for PQIP

In the proposed integrated solution of IEC 61970 and IEC 61850, the Substation Automation System (SAS) function is expanded to the EMS/SCADA side, the communication between SAS and EMS is converted to the communication inside the SAS. Hence the data transmission from the PQMD to the PQIP is based on the Manufacturing Message Specification (MMS) of IEC 61850. This solution accord with the tendency of applying the IEC 61850 to the communication and applying the IEC 61970 to the system modeling, the reconstructing workload of the existing systems is small and the practicability is high.

The following three key processes need to be implemented in the proposed solution.

(1) The PQMD need to implement IEC 61850 server which can provide the service interfaces and ICD files configuration function.

(2) The PQIP which the model is based on CIM of the IEC 61970 need to implement the IEC 61850 client to support the data access and configuration files loading of the PQMD.

(3) After the PQ monitoring data is transferred from PQMD to the PQIP, the IEC 61970 model of the PQIP need to be extended for SAS data models (IEC 61850), based on the CIM.

In the third process, based on the basic extension of CIM for substation control proposed by Electric Power Research Institute (EPRI) ${ }^{[4]}$, new entities have been defined to represent IEC 61850 substations information into IEC 61970 data models. These new entities were based on the logical nodes/common data classes description given by the IEC 61850 standard. In brief, the new entities have been defined, exploiting the description of the logical nodes of the IEC 61850-7-4 standard in order to acquire typical information and characteristics related to PQ monitoring devices. Refer to PQ monitoring function, the new entities introduced in the CIM data model are circuit breaker, metering, generator and circuit switch. The IEC 61970-7-4 standard presents logical node classes and attributes, 
represented as data classes (common data classes-IEC 61850-7-3) and not as simple types. In order to define simple types of attributes required by the database model, a merge of the information given by IEC 61850-7-3 and 7-4 has been realized. Hence, mapping with simple data types of IEC 61850-7-3 has been provided for the attributes of the logical nodes related to a specific common data class (61850-7-4), and new attributes defined by a composition of logical nodes attributes and common data classes attributes were obtained.

\section{The Architecture for Multisource Information Fusion}

Taking full advantage of massive PQ monitoring data to support the actual production process of power system is an important function of PQIP. The value of monitoring data is not limited in the PQ field, the data can be used for the distribution network fault forecast, fault location, the condition monitoring and the evaluation of the capacitor bank and circuit breaker, load monitoring and others research. At the same time the PQIP also need the useful data from other systems, such as the power grid protection configurations and their setting values from relay protection information system, the power consumption information from power marketing system. Meanwhile, the power transmission network and distribution network structure and real-time running information of the PQIP need to be in accordance with the SCADA/EMS and DEMS (Distribution network EMS).

The GID is used for providing standardized interfaces for PQ multisource information fusion. GID is a general-purpose request/reply oriented interface that supports browsing and querying of randomly associated structured data, including schema (class) and instance information. The GID standardized interface provide a lot of benefits, such as independence from the application category and application technology and middleware neutrality: as application independent, the same interfaces are used to wrap any application. This means that new wrappers do not need to be developed every time a new information source is added to the PQIP. This creates a consistent and easy-to-use integration framework by providing a unified programming model for multisource information fusion. In this paper, High-Speed Data Access (HSDA) which is designed to handle the unique requirements of exchanging high throughput data and Time Series Data Access (TSDA) which is designed for the exchange of arrays of data where each array contains the values of a single data point over time have been used for the transmission of PQ monitoring data and other data. The Generic Data Access (GDA) has been used for accessing and manipulating the CIM model of PQIP. The SCADA and EMS based on IEC 61970 implement a solution by which a CIM model server can distributed model change notices to client applications which is the PQIP in this paper. The proposed architecture for multisource information fusion is shown in Figure 2.

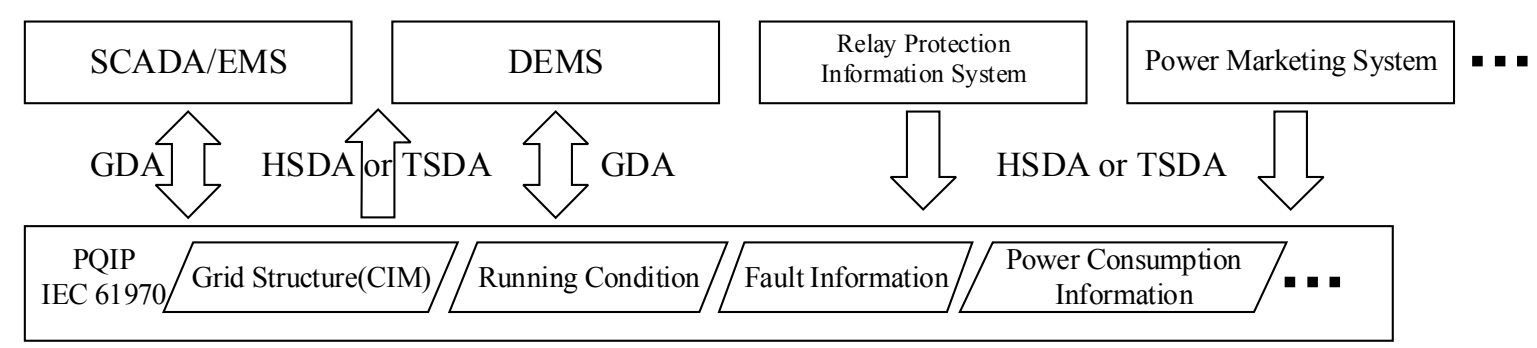

Fig. 2 The Architecture for Multisource Information Fusion based on IEC 61970

Starting with the IEC technology-independent description of PQ monitoring, a technology-dependent implementation based on web services has been provided. Web services technology refers to a structured architecture upon which computer applications can be integrated with each other by using messaging based on the eXtensible Markup Language (XML) and running over similar network architectures ${ }^{[5]}$. The aim of the web service activity is to create an integrated architecture that can reuse an existing network infrastructure, allowing clients to discover the services and data supported by a server and to provide a framework for integration development. 


\section{The Implementation of the PQIP}

The hardware architecture of the standardized PQIP for multisource information fusion is shown in Figure 3.

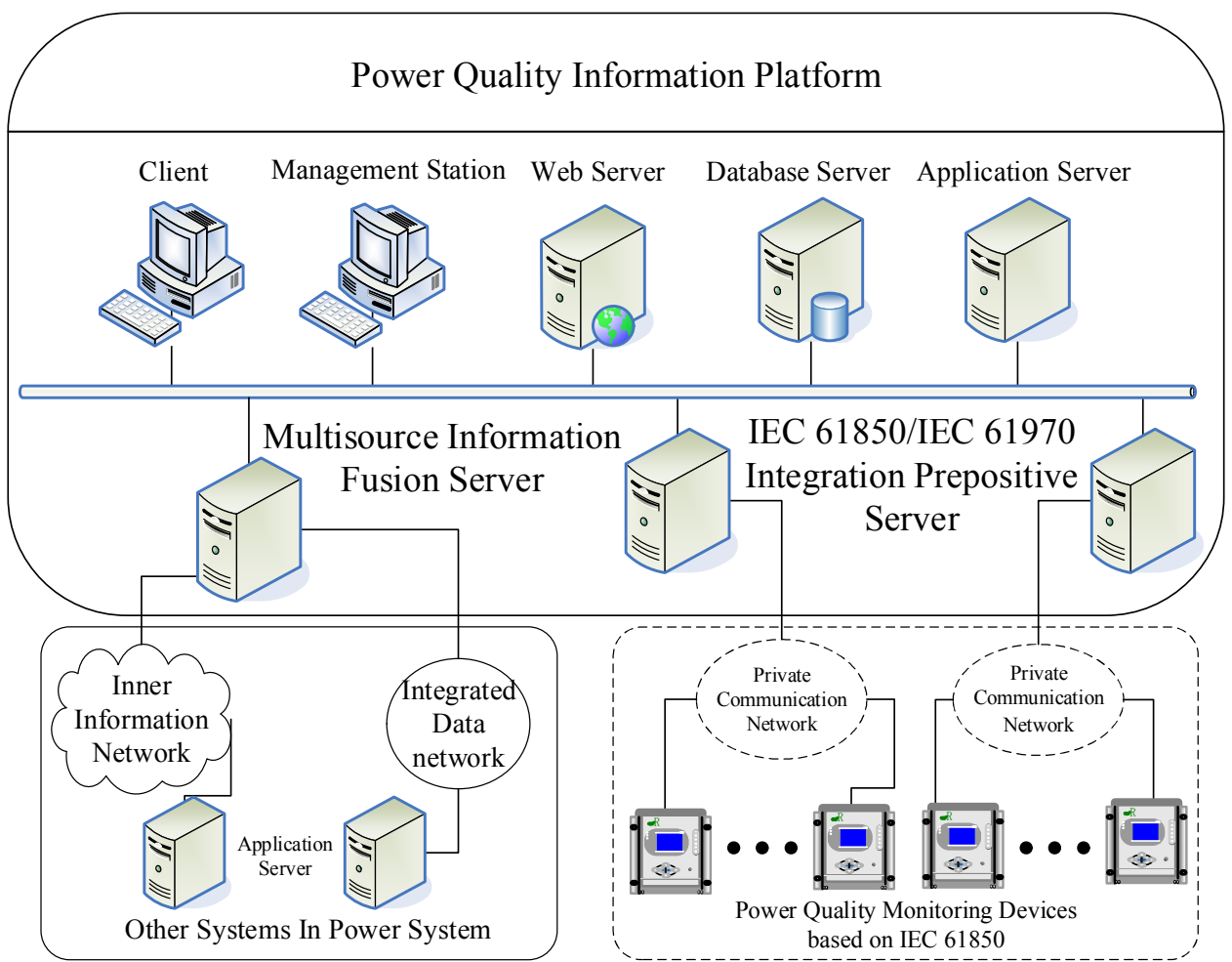

Fig. 3 The Hardware Architecture of the PQIP for Multisource Information Fusion

In the architecture, the multisource information fusion server is used to implement the Web Services for the information interaction and function interoperability based on CIM data model and CID interface of IEC 61970. The IEC 61850/IEC 61970 integration prepositive server is used to access the PQMD based on IEC 61850, the IEC 61850 client is implemented in this server and it is also responsible for changing the monitoring data based on Data Set to the CIM data model.

\section{Conclusion}

The proposed PQIP in this paper can realize functional interoperability with other systems. The massive PQ monitoring data can be taken full advantage of and the PQIP can use the grid structure and real-time for PQ analysis. The standardized PQMD based on IEC 61850 can access to the PQIP, the construction cost for both the PQSM and PQMD providers can be reduced.

\section{References:}

[1] ZHANG Yi, LIN Yan and WU Danyue. Current status and development trend of power quality monitoring system[J].Power System Protection and Control,2015,43(2):138-147

[2] ZHANG Yi, YANG Honggeng, and YE Maoqing. A SOA-Based Power Quality Management Platform[J], Advanced Materials Research, 2011(354-355):433-437.

[3] ZHANG Yi, YANG Honggeng, YE Maoqing, et al. Power quality ancillary service platform based on multi-agent[J]. Electric Power Automation Equipment, 2012, 32(12): 92-96.

[4] SISCO Inc., "Integration of substation data," 2006.

[5] ZHANG Yi, YANG Honggeng, and ZHAO Jun. Application of Web Services Dynamic Composition Based on Multi-agent in Power Quality Aided Service Platform, Proceedings of Power and Energy Engineering Conference, APPEEC2011: 1-4 